Supplement of Atmos. Chem. Phys., 21, 7321-7341, 2021

https://doi.org/10.5194/acp-21-7321-2021-supplement

(c) Author(s) 2021. CC BY 4.0 License.

(c) (1)

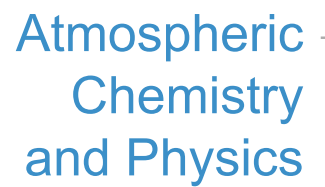

Atmospheric

and Physics

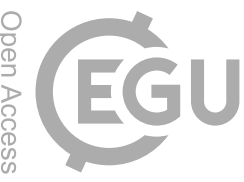

Supplement of

\title{
Source apportionment of fine organic carbon at an urban site of Beijing using a chemical mass balance model
}

\section{Jingsha Xu et al.}

Correspondence to: Zongbo Shi (z.shi@bham.ac.uk) and Roy M. Harrison (r.m.harrison@bham.ac.uk)

The copyright of individual parts of the supplement might differ from the article licence. 

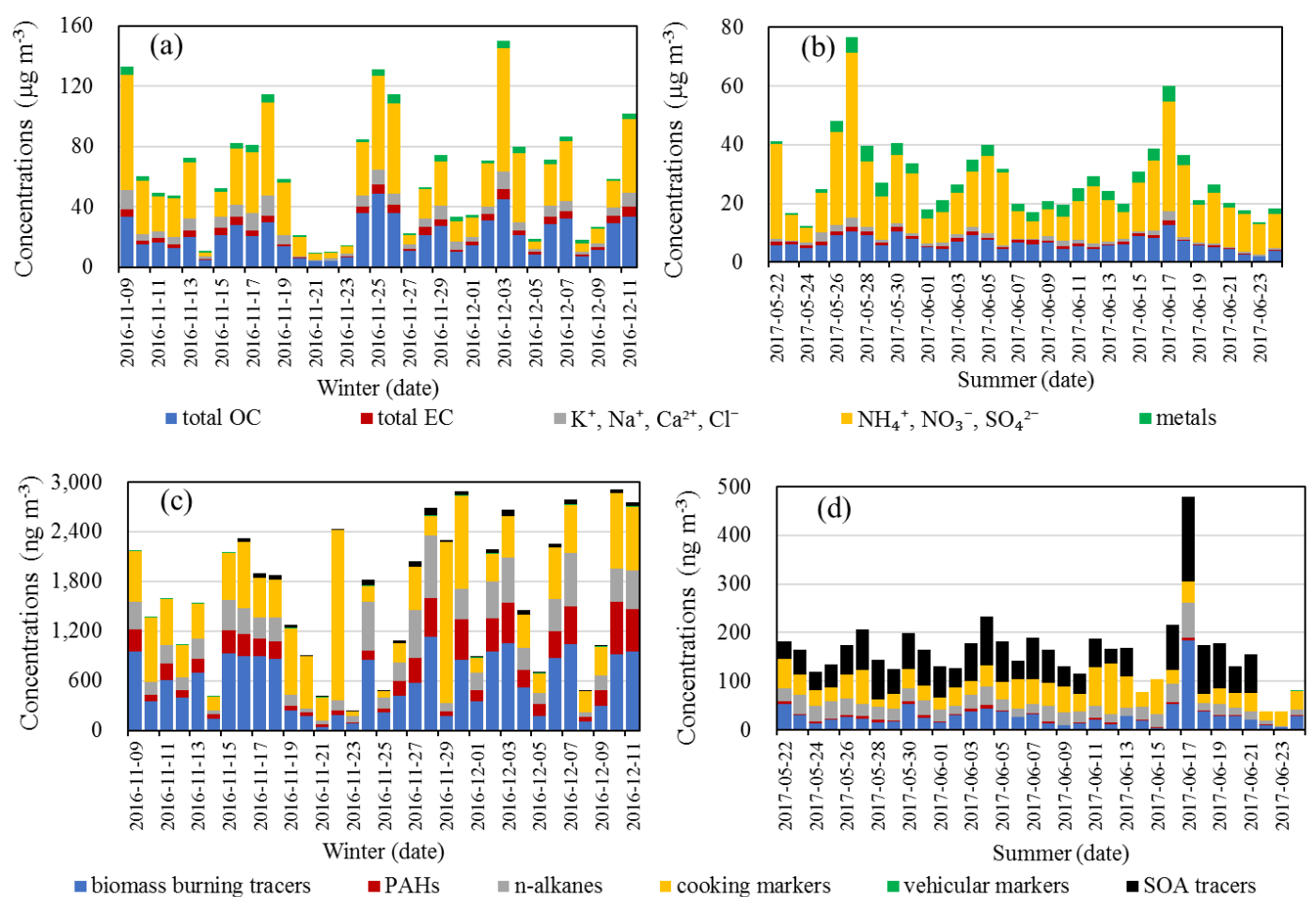

Figure S1. Stacked bar plots of the concentrations of $\mathrm{PM}_{2.5}$ components. Abbreviations: OC: organic carbon; EC: elemental carbon; PAH: polycyclic aromatic hydrocarbon; SOA: secondary organic aerosol. "Metals" is the summed concentrations of Al, Ti, V, Cr, Mn, Fe, Co, Ni, Cu, Zn, Cd, Sr, Sb, Sn, Ba, Pb; "PAH" is the summed concentrations of phenanthrene, anthracene, fluoranthene, acenaphthylne, acenaphthene, fluorene, pyrene, benzo(a)anthracene, chrysene, benzo(b)fluorene, benzo(b)fluoranthene, benzo(k)fluoranthene, benzo(a)pyrene, benzo(e)pyrene, perylene, indeno(1,2,3 cd)pyrene, dibenzo(a,h)anthracene, benzo(ghi)perylene, coronene, picence and retene; "n-alkane" is the summed concentrations of C24, C25, C26, C27, C28, C29, C30, C31, C32, C33, C34; "cooking markers" is the summed concentrations of palmitic acid, stearic acid, cholesterol; "vehicle markers" is the summed concentrations of $17 \mathrm{a}(\mathrm{H})-22,29,30$-trisnorhopane and $17 \mathrm{~b}(\mathrm{H}), 21 \mathrm{a}(\mathrm{H})$-norhopane; "SOA" is the summed concentrations of 2-methylthreitol, 2-methylerythritol, 2-methylglyceric acid, cis-2-methyl-1,3,4trihydroxy-1-butene, 3-methyl-2,3,4-trihydroxy-1-butene, trans-2-methyl-1,3,4-trihydroxy-1-butene, 3hydroxyglutaric acid(3-HGA), Pinic acid, Pinonic acid, C5-alkene triols, 2-methyltetrols, 3- MBTCA (3-Methyl-1,2,3-butanetricarboxylic Acid), beta-caryophyllinic acid, 3-acetylpentanedioic acid, 3acetylhexanedioic acid, 3-isopropylpentanedioic acid, DHOPA (2,3-dihydroxy-4-oxopentanoic acid) and 3-Hydroxy-4,4-dimethylglutaric acid. 

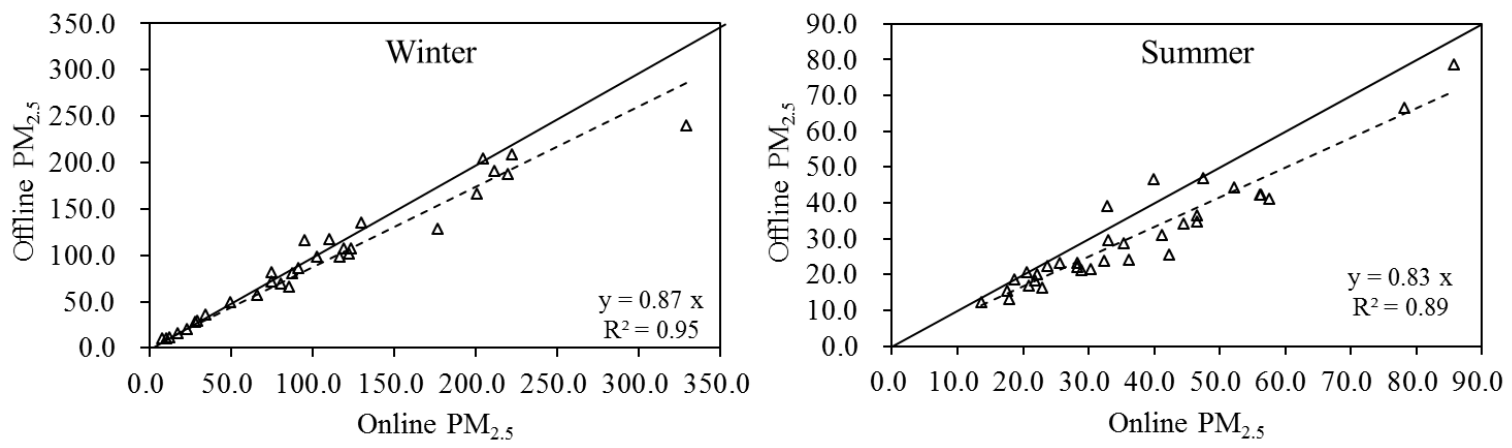

Figure S2. Regression analysis of gravimetrically measured $\mathrm{PM}_{2.5}$ (offline $\mathrm{PM}_{2.5}$ ) and online $\mathrm{PM}_{2.5}$ in winter and summer at IAP, Beijing
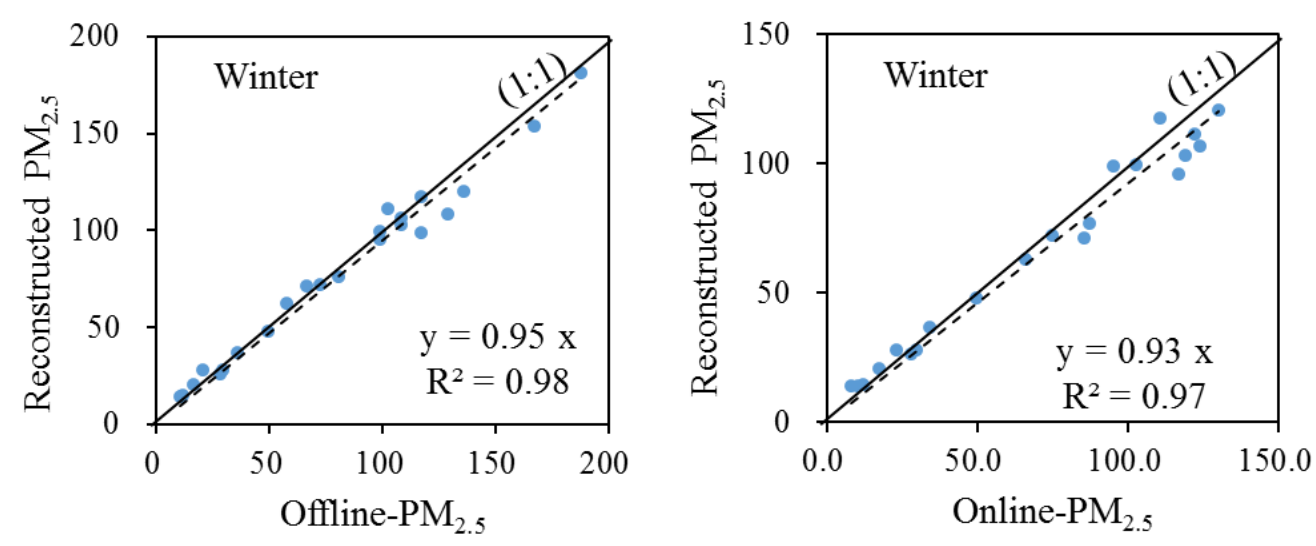

Figure S3. Regression results between reconstructed $\mathrm{PM}_{2.5}$ and offline/online $\mathrm{PM}_{2.5}$ by chemical mass closure method in winter excluding outliers.
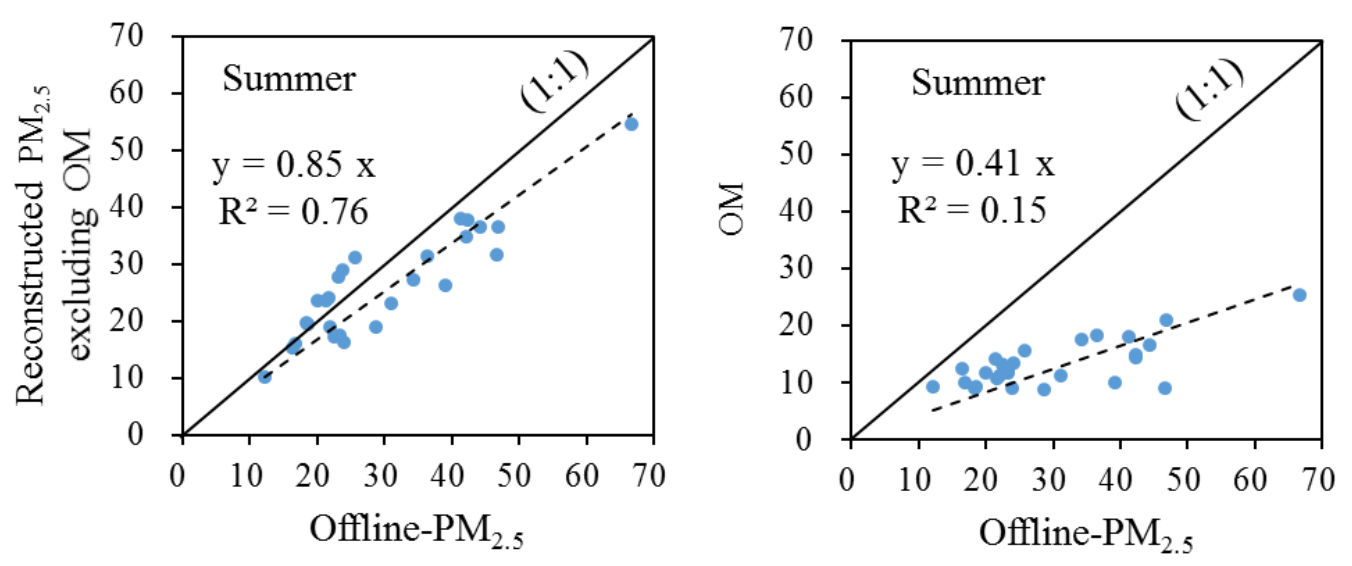

Figure S4. Regression results between inorganics (reconstructed $\mathrm{PM}_{2.5}$ excluding $\mathrm{OM}$ ) and $\mathrm{OM}$ with offline-PM $\mathrm{PM}_{2.5}$ by chemical mass closure method in winter excluding outliers. 

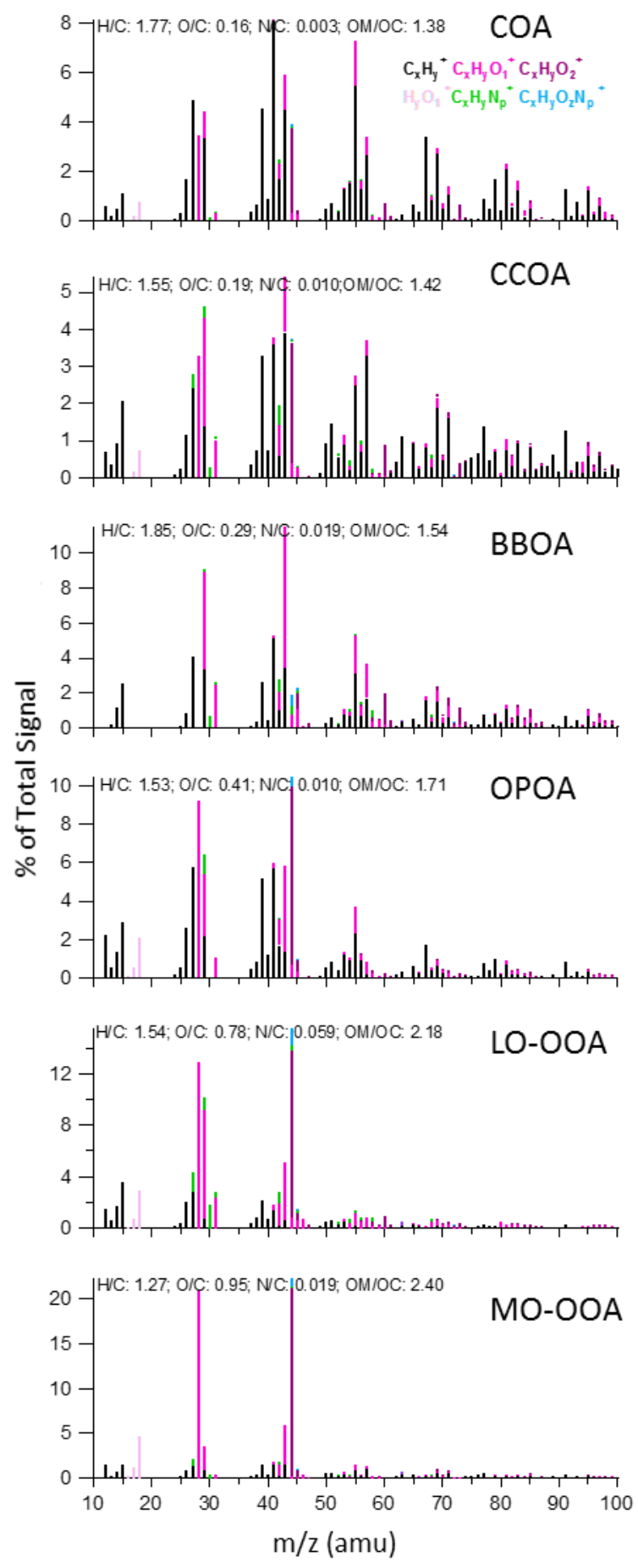

Figure S5. Source profiles of AMS-PMF factors in winter 

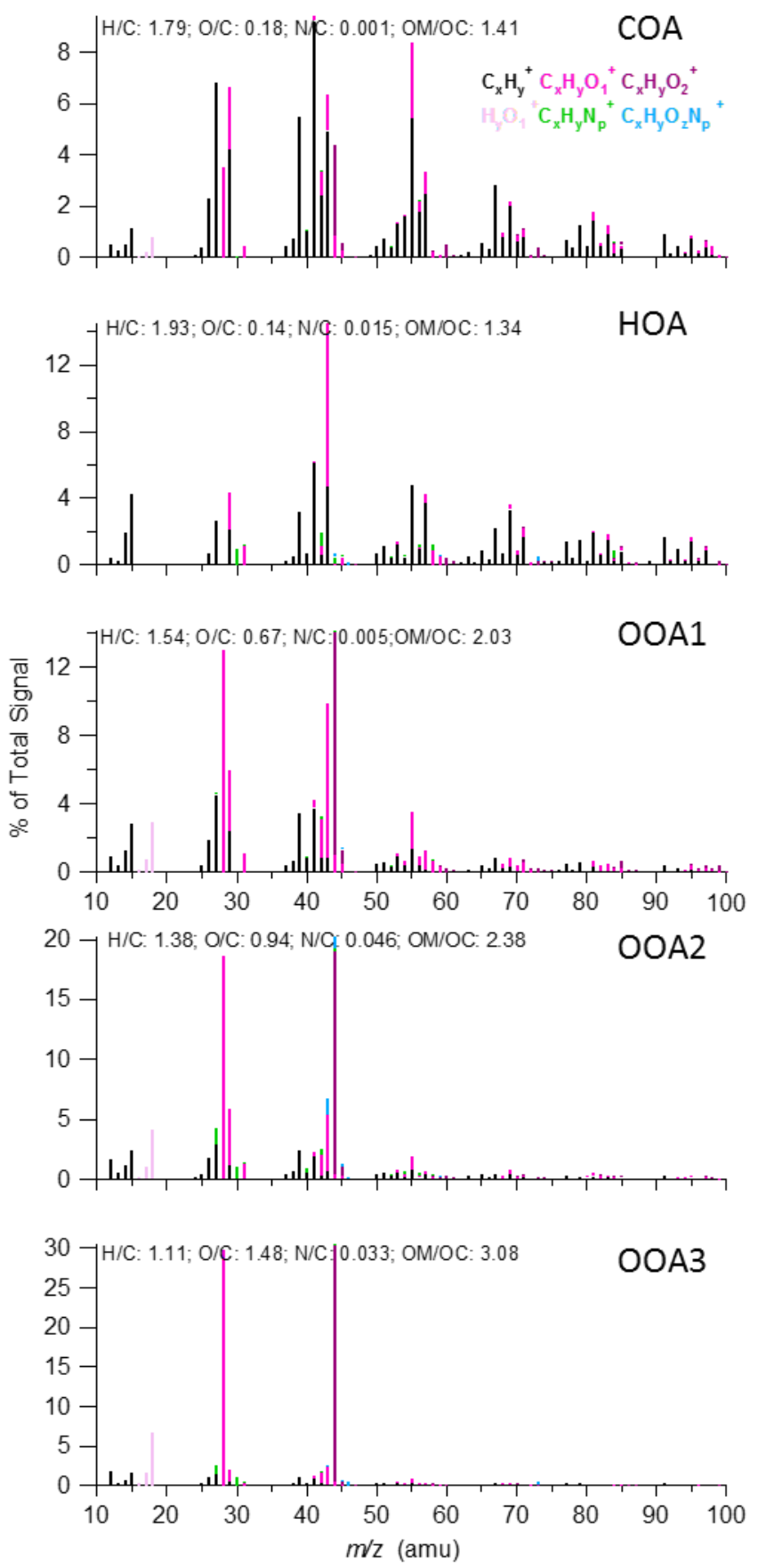

Figure S6. Source profiles of AMS-PMF factors in summer 


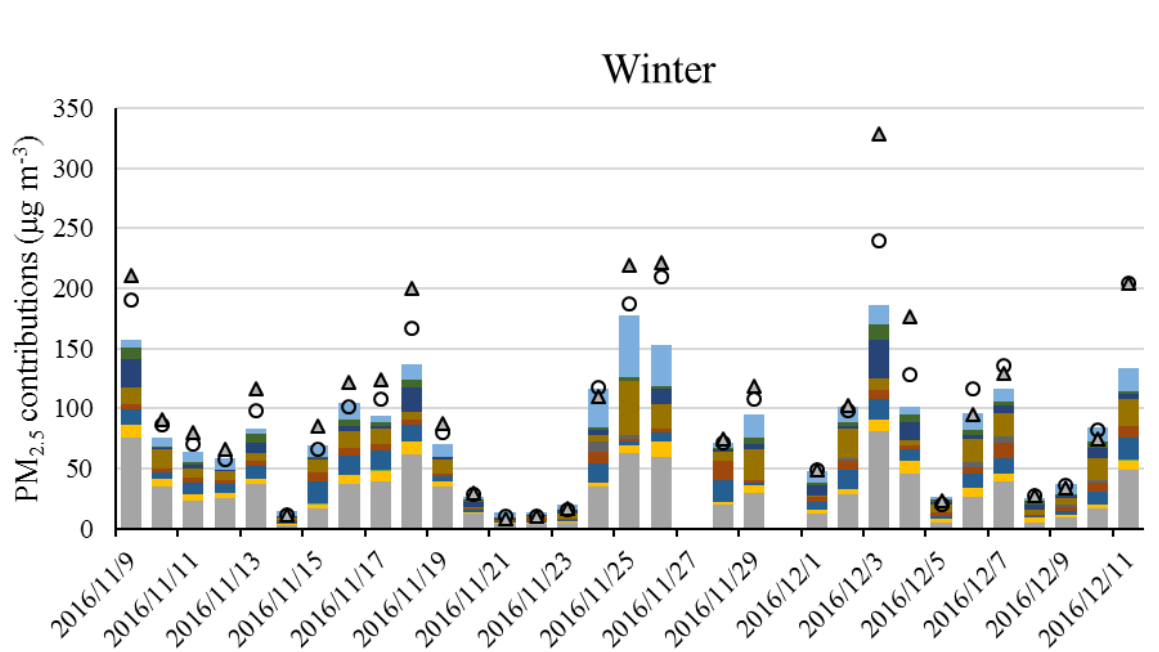

$\square$ SNA

- Geological minerals

- Vegetative detritus

- Biomass burning

- Gasoline vehicles

- Diesel vehicles

- Industrial CC

- Residential CC

- Cooking

Other OM

○ Offline $\mathrm{PM}_{2.5}$

$\Delta$ Online $\mathrm{PM}_{2 \cdot 5}$

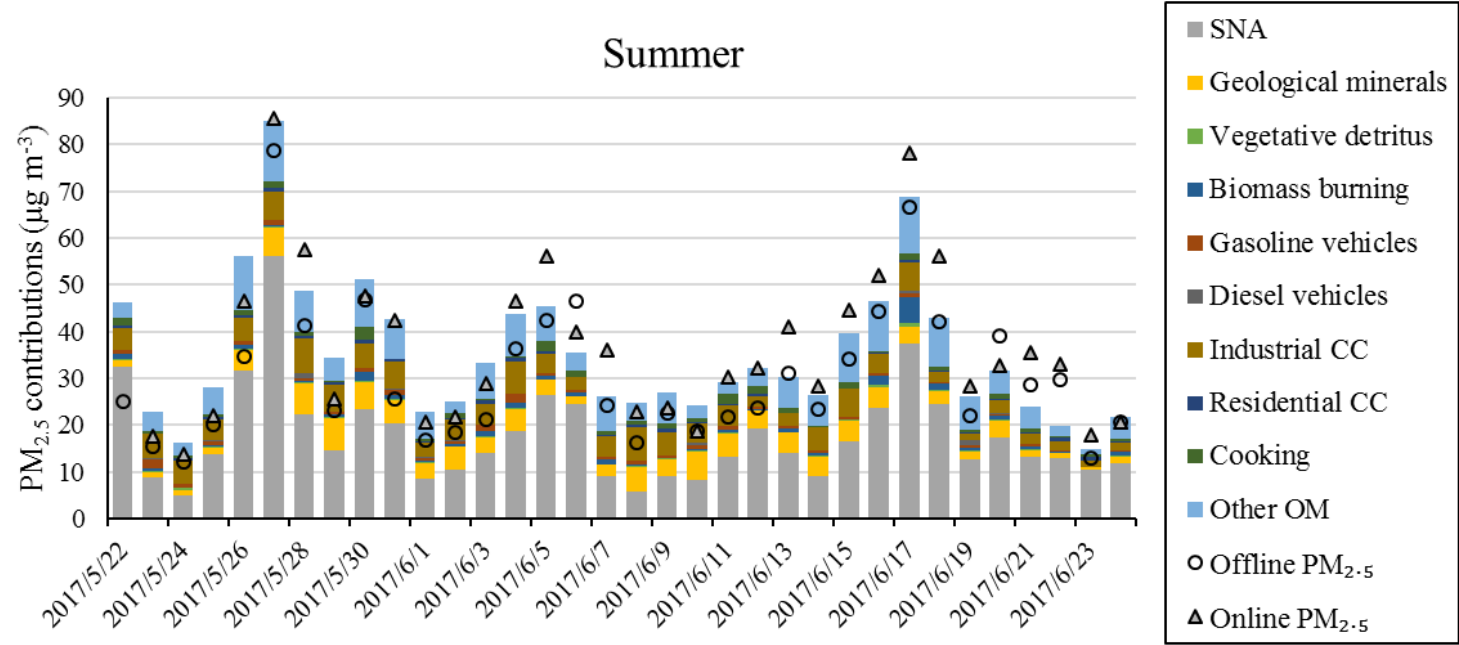

Figure S7. Daily $\mathrm{PM}_{2.5}$ source contribution estimates from the CMB model 
Table S1. Concentrations (mean \pm SD (min-max), $\mu \mathrm{g} \mathrm{m}^{-3}$ ) of chemical components in $\mathrm{PM}_{2.5}$ by applying chemical mass closure method

\begin{tabular}{|c|c|c|}
\hline Component & Winter & Summer \\
\hline $\mathrm{NO}_{3}^{-}$ & $12.49 \pm 9.38(0.87-34.63)$ & $7.16 \pm 4.94(1.53-26.12)$ \\
\hline $\mathrm{SO}_{4}^{2-}$ & $8.53 \pm 7.05(1.27-24.21)$ & $6.87 \pm 3.96(1.96-19.48)$ \\
\hline $\mathrm{NH}_{4}^{+}$ & $8.24 \pm 5.63(0.50-22.62)$ & $3.62 \pm 3.14(0.08-14.83)$ \\
\hline $\mathrm{Cl}^{-}$ & $3.80 \pm 2.32(0.00-8.73)$ & $0.47 \pm 0.42(0.12-1.96)$ \\
\hline $\mathrm{K}^{+}$ & $1.30 \pm 1.01(0.15-3.80)$ & $0.37 \pm 0.35(0.11-2.05)$ \\
\hline $\mathrm{Na}^{+}$ & $0.42 \pm 0.24(0.09-0.93)$ & $0.20 \pm 0.16(0.03-0.73)$ \\
\hline Geological minerals & $5.33 \pm 3.25(1.03-12.72)$ & $3.52 \pm 1.79(0.65-6.99)$ \\
\hline Total other elements & $0.53 \pm 0.35(0.12-1.29)$ & $0.35 \pm 0.20(0.07-1.00)$ \\
\hline $\mathrm{EC}$ & $3.5 \pm 2.0(0.3-6.6)$ & $0.9 \pm 0.4(0.2-1.7)$ \\
\hline $\mathrm{OM}$ & $37.7 \pm 21.5(6.9-85.4)$ & $12.9 \pm 4.7(3.6-25.4)$ \\
\hline Bound water-Offline & $4.0 \pm 3.7(0.2-13.4)$ & $2.8 \pm 1.4(0.8-7.3)$ \\
\hline Reconstructed $\mathrm{PM}_{2.5}$-Offline & $83.4 \pm 53.6(14.0-202.1)$ & $39.4 \pm 13.0(19.6-80.0)$ \\
\hline Offline $\mathrm{PM}_{2.5}$ & $88.6 \pm 63.6(10.3-239.9)$ & $30.0 \pm 12.7(12.2-66.7)$ \\
\hline Bound water-Online & $4.5 \pm 4.1(0.3-14.9)$ & $3.5 \pm 1.8(0.9-9.1)$ \\
\hline Reconstructed $\mathrm{PM}_{2.5}$-Online & $83.8 \pm 53.9(14.0-203.7)$ & $40.1 \pm 13.4(19.8-81.8)$ \\
\hline Online $\mathrm{PM}_{2.5}$ & $99.7 \pm 79.1(8.1-328.7)$ & $36.4 \pm 14.9(13.7-78.1)$ \\
\hline
\end{tabular}

Note: 5 samples in winter and 7 samples in summer were not included for the calculation of bound water and reconstructed $\mathrm{PM}_{2.5}$ due to insufficient ions and $\mathrm{NH}_{3}$ data. These samples were excluded for the calculation of average online and offline $\mathrm{PM}_{2.5}$ in this table as well for better comparison.

Table S2. Annual average primary OC emissions (Unit: tonne) in Beijing from the 2016 and 2017 Multiresolution Emission Inventory for China (MEIC)

\begin{tabular}{cccc}
\hline Sector & Fuel & 2016 & 2017 \\
\hline Power & Coal & 0 & 0 \\
Power & Oil & 0 & 0 \\
Power & Natural Gas & 0 & 0 \\
Industry & Coal & 0 & 0 \\
Industry & Oil & 538 & 583 \\
Industry & Natural Gas & 0 & 0 \\
Industry & Process & 1161 & 1083 \\
Residential & Coal & 6687 & 4312 \\
Residential & Oil & 24 & 23 \\
Residential & Natural Gas & 0 & 0 \\
Residential & Biofuel & 5548 & 4993 \\
Transportation & Oil & 1059 & 1026 \\
\hline Total & & 15017 & 12020 \\
\hline
\end{tabular}




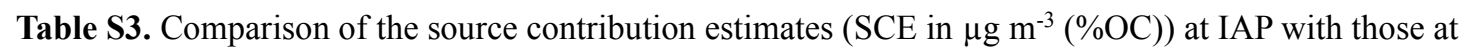
a rural site in Beijing- Pinggu

\begin{tabular}{|c|c|c|c|c|}
\hline & \multicolumn{2}{|c|}{ IAP (Urban) (This study) } & \multicolumn{2}{|l|}{ Pinggu (Rural) } \\
\hline & Winter (31 days) & Summer (34 days) & Winter (14 days) & Summer (6 days) \\
\hline $\mathrm{OC}$ & $21.5 \pm 12.3$ & $6.4 \pm 2.3$ & $36.5 \pm 29.3$ & $10.7 \pm 4.9$ \\
\hline OC explained & $75.7 \pm 11.0 \%$ & $56.1 \pm 11.3 \%$ & $69.1 \pm 7.1 \%$ & $63.4 \pm 12.6 \%$ \\
\hline Vegetative detritus & $0.1 \pm 0.1(0.5 \pm 0.4 \%)$ & $0.1 \pm 0.1(1.7 \pm 0.8 \%)$ & $1.5 \pm 3.0(2.8 \pm 3.4 \%)$ & $0.3 \pm 0.3(2.1 \pm 1.4 \%)$ \\
\hline Biomass burning & $3.8 \pm 2.6(17.4 \pm 8.7 \%)$ & $0.3 \pm 0.4(4.8 \pm 3.4 \%)$ & $6.8 \pm 5.6(18.1 \pm 3.4 \%)$ & $1.1 \pm 0.6(10.7 \pm 2.6 \%)$ \\
\hline Gasoline & $2.0 \pm 1.6(10.2 \pm 6.6 \%)$ & $0.3 \pm 0.2(4.9 \pm 2.2 \%)$ & $1.0 \pm 0.9(3.4 \pm 1.6 \%)$ & $0.1 \pm 0.0(1.3 \pm 0.6 \%)$ \\
\hline Diesel & $0.5 \pm 1.2(1.9 \pm 3.7 \%)$ & $0.1 \pm 0.2(1.2 \pm 2.5 \%)$ & $6.2 \pm 6.0(13.7 \pm 6.0 \%)$ & $0.6 \pm 0.3(6.2 \pm 4.8 \%)$ \\
\hline Industrial CC & $4.9 \pm 4.1(22.0 \pm 11.2 \%)$ & $1.8 \pm 0.7(29.0 \pm 9.0 \%)$ & $3.2 \pm 2.6(10.2 \pm 5.7 \%)$ & $3.8 \pm 2.5(34.1 \pm 11.0 \%)$ \\
\hline Residential CC & $2.6 \pm 3.1(12.5 \pm 10.2 \%)$ & $0.2 \pm 0.1(3.3 \pm 3.5 \%)$ & $5.7 \pm 4.3(19.0 \pm 12.4 \%)$ & $0.4 \pm 0.2(4.2 \pm 1.8 \%)$ \\
\hline Cooking & $2.2 \pm 2.1(10.6 \pm 7.3 \%)$ & $0.7 \pm 0.4(11.1 \pm 7.1 \%)$ & $0.5 \pm 0.5(2.0 \pm 2.3 \%)$ & $0.5 \pm 0.4(4.9 \pm 3.9 \%)$ \\
\hline Other OC & $5.3 \pm 4.9(24.8 \pm 12.1 \%)$ & $2.9 \pm 1.5(43.9 \pm 11.4 \%)$ & $11.7 \pm 10.4(30.9 \pm 7.1 \%)$ & $3.9 \pm 2.3(36.6 \pm 12.6 \%)$ \\
\hline
\end{tabular}

Table S4. The ratios of OC/PM 2.5 (or OM/OC) for different sources

\begin{tabular}{lll}
\hline & $\mathrm{OC} / \mathrm{PM}_{2.5}$ & Reference \\
\hline Straw burning & 0.546 & (Zhang et al., 2007) \\
\hline Wood burning & 0.3 & (Wang et al., 2009) \\
\hline Cooking & $1.4(\mathrm{OM} / \mathrm{OC})$ & (Zhao et al., 2007) \\
\hline Light-duty gasoline cars & 0.317 & \\
Heavy-duty gasoline cars & 0.549 & (Cai et al., 2017) \\
Diesel cars & 0.569 & \\
\hline Vegetative detritus & $2.1(\mathrm{OM} / \mathrm{OC})$ & (Bae et al., 2006b) \\
\hline Anthracite & 0.446 & (Zhang et al., 2008) \\
Bituminite & 0.403 & \\
Coal briquette & 0.432 & (Bae et al., 2006a) \\
\hline Secondary organic aerosol & $2.17(\mathrm{OM} / \mathrm{OC})$ & (Zhang et al., 2005) \\
\hline Oxygenated OA & $2.2(\mathrm{OM} / \mathrm{OC})$ & (Aiken et al., 2008) \\
\hline Oxygenated OA & $1.85 \sim 2.3(\mathrm{OM} / \mathrm{OC})$ & \\
\hline
\end{tabular}


Table S5. Reconstructed source contributions (mean \pm SD of the daily values) and their relative abundance (mean $\pm \mathrm{SD}$ of the daily values) in reconstructed $\mathrm{PM}_{2.5}$ mass in urban Beijing

\begin{tabular}{|c|c|c|c|c|c|}
\hline & \multicolumn{2}{|c|}{ Mass concentrations $\left(\mu \mathrm{g} \mathrm{m}^{-3}\right)$} & & \multicolumn{2}{|c|}{$\begin{array}{l}\text { Mass concentrations/ } \\
\text { Reconstructed mass }(\%)\end{array}$} \\
\hline & Winter & Summer & & Winter & Summer \\
\hline SNA & $30.5 \pm 21.8$ & $17.7 \pm 10.5$ & SNA/ RM & $34.1 \pm 9.7$ & $48.5 \pm 11.9$ \\
\hline Geological minerals & $5.3 \pm 3.2$ & $3.5 \pm 1.8$ & Geological minerals/ RM & $7.0 \pm 3.0$ & $10.4 \pm 5.5$ \\
\hline Vegetative detritus & $0.2 \pm 0.2$ & $0.2 \pm 0.2$ & Vegetative detritus/ RM & $0.3 \pm 0.2$ & $0.7 \pm 0.4$ \\
\hline Biomass burning & $8.9 \pm 6.2$ & $0.8 \pm 0.9$ & Biomass burning/ RM & $11.0 \pm 5.9$ & $2.1 \pm 1.5$ \\
\hline Gasoline vehicles & $4.7 \pm 3.6$ & $0.7 \pm 0.4$ & Gasoline vehicles/ RM & $6.5 \pm 4.8$ & $2.2 \pm 1.4$ \\
\hline Diesel vehicles & $0.9 \pm 2.0$ & $0.1 \pm 0.3$ & Diesel vehicles/ RM & $1.0 \pm 2.0$ & $0.4 \pm 0.9$ \\
\hline Industrial $\mathrm{CC}$ & $11.6 \pm 9.7$ & $4.3 \pm 1.7$ & Industrial CC/ RM & $13.8 \pm 7.3$ & $13.2 \pm 6.1$ \\
\hline Residential CC & $6.1 \pm 7.3$ & $0.4 \pm 0.3$ & Residential CC/ RM & $7.6 \pm 5.7$ & $1.4 \pm 1.1$ \\
\hline Cooking & $3.1 \pm 3.0$ & $0.9 \pm 0.6$ & Cooking/ RM & $3.9 \pm 2.5$ & $2.8 \pm 1.6$ \\
\hline Other OM & $11.7 \pm 10.8$ & $6.5 \pm 3.2$ & Other OM/ RM & $14.8 \pm 7.9$ & $18.3 \pm 5.9$ \\
\hline $\begin{array}{l}\text { Reconstructed mass } \\
(\mathrm{RM})\end{array}$ & $83.1 \pm 49.0$ & $35.1 \pm 15.0$ & & & \\
\hline Offline $\mathrm{PM}_{2.5}$ mass & $105.0 \pm 77.4$ & $36.5 \pm 17.0$ & $\begin{array}{l}\text { Reconstructed mass/ } \\
\text { Offline } \mathrm{PM}_{2.5} \text { mass }\end{array}$ & $96.6 \pm 17.6$ & $121.7 \pm 26.6$ \\
\hline Online $\mathrm{PM}_{2.5}$ mass & $94.8 \pm 64.4$ & $30.2 \pm 14.8$ & $\begin{array}{l}\text { Reconstructed mass/ } \\
\text { Offline } \mathrm{PM}_{2.5} \text { mass }\end{array}$ & $91.9 \pm 24.1$ & $99.0 \pm 19.1$ \\
\hline
\end{tabular}

\section{References}

Aiken, A. C., DeCarlo, P. F., Kroll, J. H., Worsnop, D. R., Huffman, J. A., Docherty, K. S., Ulbrich, I. M., Mohr, C., Kimmel, J. R., Sueper, D., Sun, Y., Zhang, Q., Trimborn, A., Northway, M., Ziemann, P. J., Canagaratna, M. R., Onasch, T. B., Alfarra, M. R., Prevot, A. S. H., Dommen, J., Duplissy, J., Metzger, A., Baltensperger, U., and Jimenez, J. L.: O/C and OM/OC Ratios of Primary, Secondary, and Ambient Organic Aerosols with High-Resolution Time-of-Flight Aerosol Mass Spectrometry, Environ. Sci. Technol., 42, 4478-4485, 10.1021/es703009q, 2008.

Bae, M.-S., Demerjian, K. L., and Schwab, J. J.: Seasonal estimation of organic mass to organic carbon in PM2.5 at rural and urban locations in New York state, Atmospheric Environment, 40, 7467-7479, https://doi.org/10.1016/j.atmosenv.2006.07.008, 2006a.

Bae, M.-S., Schauer, J. J., and Turner, J. R.: Estimation of the Monthly Average Ratios of Organic Mass to Organic Carbon for Fine Particulate Matter at an Urban Site, Aerosol Sci. Technol., 40, 1123-1139, 10.1080/02786820601004085, 2006 b.

Cai, T., Zhang, Y., Fang, D., Shang, J., Zhang, Y., and Zhang, Y.: Chinese vehicle emissions characteristic testing with small sample size: Results and comparison, Atmospheric Pollution Research, 8, 154-163, https://doi.org/10.1016/j.apr.2016.08.007, 2017.

Wang, Q., Shao, M., Zhang, Y., Wei, Y., Hu, M., and Guo, S.: Source apportionment of fine organic aerosols in Beijing, Atmos. Chem. Phys., 9, 8573-8585, 10.5194/acp-9-8573-2009, 2009.

Zhang, Q., Worsnop, D. R., Canagaratna, M. R., and Jimenez, J. L.: Hydrocarbon-like and oxygenated organic aerosols in Pittsburgh: insights into sources and processes of organic aerosols, Atmospheric 
Chemistry And Physics, 5, 3289-3311, 10.5194/acp-5-3289-2005, 2005.

Zhang, Y.-x., Shao, M., Zhang, Y.-h., Zeng, L.-m., He, L.-y., Zhu, B., Wei, Y.-j., and Zhu, X.-1.: Source profiles of particulate organic matters emitted from cereal straw burnings, Journal of Environmental Sciences, 19, 167-175, https://doi.org/10.1016/S1001-0742(07)60027-8, 2007.

Zhang, Y., Schauer, J. J., Zhang, Y., Zeng, L., Wei, Y., Liu, Y., and Shao, M.: Characteristics of Particulate Carbon Emissions from Real-World Chinese Coal Combustion, Environ. Sci. Technol., 42, 5068-5073, 10.1021/es7022576, 2008.

Zhao, Y., Hu, M., Slanina, S., and Zhang, Y.: Chemical Compositions of Fine Particulate Organic Matter Emitted from Chinese Cooking, Environ. Sci. Technol., 41, 99-105, 10.1021/es0614518, 2007. 